http://doi.org/10.15359/ree.13-2.7

\title{
Currículo en el nivel preescolar costarricense: El Ciclo Materno Infantil (CMI)
}

\author{
Ana Lucía Chaves Álvarez. \\ División de Educación Básica, \\ Centro de Investigación y Docencia en Educación, Universidad Nacional \\ Heredia, Costa Rica
}

Recibido 20 de abril de 2009 • Aprobado 14 de setiembre de 2009

"El aprendizaje no tiene lugar mediante la transmisión o por reproducción, sino que más bien se configura como un proceso de construcción de razones, de los porqués, de los significados, del sentido de las cosas, de los otros, de la naturaleza, de los acontecimientos, de la realidad, de la vida".

Reggio Emilia, Carlina Rinldi, citadas por D’Angelo y Medina,1999

Resumen. Este artículo aborda la conceptuación y la descripción del Ciclo Materno Infantil como parte del nivel de preescolar del sistema educativo formal costarricense. Con el propósito de tener una visión más amplia de este Ciclo, se hace un repaso por el concepto de currículo, así como de los elementos que lo conforman, y, luego, se detallan específicamente los aspectos relacionados con el currículo de preescolar costarricense del Ciclo Materno Infantil, tomando como punto de partida el Programa de Estudio que se elaboró para atender este Ciclo en el sistema educativo de Costa Rica.

Palabras clave. currículo, educación preescolar, docentes, niños y niñas, Ciclo Materno Infantil, Programa de Estudio del Ciclo Materno Infantil.

1 Licenciada en Pedagogía con Énfasis en Educación Preescolar, graduada en la Universidad Nacional. Tiene experiencia docente en instituciones educativas costarricenses, en las que ha trabajado con niños y niñas del nivel de preescolar y de primaria. Actualmente, labora en la División de Educación Básica del Centro de Investigación y Docencia en Educación, como académica de la carrera de Educación Preescolar y como miembro del equipo del Proyecto de Acreditación.

Correo electrónico: analuciachaves@hotmail.com 
Abstract. The present article tackles the conceptualization and description of the Infantile Maternal Cycle as p The present article tackles the conceptualization and description of the Infantile Maternal Cycle as part of the preschool level of the Costa Rican educational system. With the intention of having a wider vision of this Cycle, a revision is done for the curriculum concept, as well as, for the elements that shape it, and then there is a detailed description of specific aspects related to the preschool Costa Rican curriculum of the Infantile Maternal Cycle, taking as a starting point the Program of Study that was designed to attend the above Cycle in the Costa Rican educational system.

Key words. Curriculum, Pre- school education, teachers, children, Infantile Maternal Cycle, Program of the Infantile Maternal Cycle.

\section{Introducción}

Sin duda alguna, una manera de hacer que la educación preescolar sea significativa y útil, es partiendo de las características, intereses y necesidades de la población infantil. Precisamente en el currículo es en donde se pueden concretar esos tres aspectos. Este documento surge de la investigación titulada "Análisis de la congruencia entre el Programa de Estudio para el Ciclo Materno Infantil del Ministerio de Educación Pública y las características, intereses y necesidades de los niños y las niñas que asisten a los grupos Interactivos II de dos jardines infantiles públicos del área metropolitana" (Chaves, 2004). Lo que se pretendió fue hacer un recorrido por el Ciclo Materno Infantil (CMI), como parte del currículo del sistema educativo costarricense. Para lograr esto, en primer lugar, se detallan los aspectos relacionados con el currículo desde una perspectiva en general, y, luego, se ahonda en aspectos específicos de este primer Ciclo de la educación preescolar costarricense.

\section{Consideraciones teóricas generales del currículo}

Es pertinente anotar que existen muchas definiciones en torno al concepto de currículo. Es fácil deducir que cada autor construye una definición de currículo de acuerdo con las ideas y las experiencias que tenga. Como se puede apreciar, las definiciones que se incluyen tienen algunos aspectos en común, pero también presentan algunas diferencias entre sí; en general, todas ellas abordan aspectos importantes de tomar en cuenta para comprender mejor este concepto.

Una definición de currículo que se considera importante conocer es la que plantea el Ministerio de Educación Pública (MEP) (2001), en el Programa de Estudio del Ciclo Materno Infantil (PECMI). Ésta se enfoca hacia los y las estudiantes, la investigación, el contexto y los procesos de construcción de aprendizaje. Para el MEP (2001),

(...) se define currículo como un proceso de investigación,que comprende al individuo (formación integral), a su contexto (formación integrada) y la relación entre ambos (formación integradora) para mejorar los procesos de construcción de conocimientos, en la formación de la persona y el mejoramiento de su calidad de vida, así como el funcionamiento de la institución educativa y la comunidad. (p. 53)

De esta definición se deduce que al currículo se le asigna una función, la cual consiste, en forma general, en contribuir al desarrollo integral de las personas. De ella se desprende que para poder lograr que el currículo contribuya al desarrollo integral de los sujetos, es indispensable realizar procesos investigativos, que permitan conocer a cuáles individuos va dirigido y cómo es el contexto en el que éstos se desenvuelven. 
El MEP (2001) agrega que esa conceptuación del currículo “ (...) implica que éste se reconstruye según los cambios, necesidades y expectativas de las personas a quienes va dirigido, del contexto sociocultural y los resultados en las investigaciones en (...) la educación" (p. 53). Lo anterior es muy importante, ya que de este modo, se espera la renovación del currículo para responder, de una forma efectiva, a los requerimientos de los individuos y de las sociedades a las que pertenecen.

Esta definición, al igual que las siguientes, hacen referencia a la conceptuación del currículo, tal como se mencionó al inicio, o sea, que cada autor imprime su sello personal y enfatiza los aspectos que considera más relevantes, en el momento de definirlo. Se incluyen varias definiciones de este término, para hacer ver al lector, la gran variedad que existe. Es importante rescatar, que todas las definiciones son válidas y todas brindan pistas importantes que permiten conocer, con mayor amplitud, lo que significa esta idea. Una sola definición sería insuficiente para comprender todo lo que el currículo implica.

Es pertinente anotar, después de haber aclarado lo anterior, que el MEP (2009) plantea una definición de currículo que ofrece una visión amplia de este concepto, ya que no es concebido únicamente como una guía de la práctica educativa, sino que implica todo el proceso educativo como tal y, además, todos los factores que en él intervienen. De este modo, el MEP indica que el currículo es "la integración sistemática de todos los componentes que inciden en el quehacer educativo, para el desarrollo integral de la persona y de la sociedad, en armonía con la naturaleza y consecuente con un desarrollo sostenible" (p. 31).

Por otra parte, Bolaños y Molina (2001) hacen referencia al currículo y argumentan que por medio de él se pueden lograr las intenciones de la educación. Estos autores señalan que

Las intencionalidades de la educación, en términos de los resultados que se pretende alcanzar en cuanto a la formación del ciudadano y el tipo de sociedad, se concretan mediante el currículo que actúa (...) como un proceso operativo, en el que entran en juego un conjunto de elementos (actores sociales, objetivos, recursos, etc.) que interactúan para alcanzar esas intencionalidades educativas. (p. 24)

Si se toman en cuenta las argumentaciones anteriores, podría decirse que el currículo, involucra a todos los participantes de los procesos educativos, con el propósito de que la educación sea significativa para las y los estudiantes y, en general, para toda la sociedad. Éste es uno de los principales propósitos de la educación, el cual se puede alcanzar, mediante el diseño de un currículo, como el que plantean los autores citados.

Peralta (2007), investigadora de gran trascedencia en el ámbito de la educación preescolar, también, hace alusión a este concepto. Esta autora define el currículo de la siguiente forma:

Todas las vivencias y aprendizajes significativos que han sido experienciados por los párvulos y adultos, como resultante de la selección y organización consistente de un conjunto de factores humanos, materiales y técnicos, que han sido generados por una comunidad educativa, desde el Jardín Infantil como institución educativa sistemática. (p. 6)

De la definición anterior, se desprende que el currículo es concebido como un proceso significativo. Precisamente, para poder lograr una educación relevante, tal y como se evidencia en el planteamiento de Peralta (2007), es importante que el currículo sea planificado en forma adecuada, para que tenga trascendencia en la vida de los individuos que participan en él. 
Asimismo, Grundy (1998) hace algunos planteamientos relacionados con el currículo. Para esta autora "(...) no es un concepto, sino una construcción cultural. Es decir, no se trata de un concepto abstracto que tenga alguna existencia aparte de y antecedente a la experiencia humana. Es, en cambio, una forma de organizar un conjunto de prácticas educativas humanas" (p. 20).

Salta a la vista, que para Grundy (1998), el currículo está determinado y adquiere sentido, en el contexto en el cual se desarrolla. Según esta autora, el currículo es considerado como tal, en la medida en que parta de lo que los sujetos son y del contexto en el que se desenvuelven; se desprende, entonces, que un simple discurso plasmado en un papel, que intente guiar la práctica educativa, pero que no tome en cuenta lo anterior, no podría ser catalogado como currículo.

Además de los autores ya citados, Quesada, Cedeño y Zamora (2001) también hacen mención a lo que significa el término currículo. Estos autores intentan conceptualizar el currículo en un marco amplio, en el que las personas que participan en él juegan un papel de gran relevancia. Postulan que el currículo, es el que permite el cambio y la transformación en los procesos educativos. Cabe señalar que eso ocurrirá, siempre y cuando el currículo se renueve constantemente, y los encargados de su diseño busquen ideas innovadoras que, en realidad, promuevan un cambio útil en la educación.

Además, Quesada et al. (2001) argumentan que dentro del currículo, todos sus participantes deben tener una constante interacción. Al respecto, consideran que

La forma de entender el currículo (...) corresponde a una construcción social en la que el sujeto del currículo es un ser social, histórico, concreto y reflexivo, que posee un sistema de creencias; donde existe una relación dialógica entre el docente y el estudiante, donde los alumnos interactúan con otros alumnos, con los profesores y con su propio pensamiento.

Precisamente, ambos actores, docentes y estudiantes, convierten en práctica cotidiana un curriculum y le imprimen diversos significados y sentidos; a su vez, lo transforman con el aporte de la experiencia de aula, la investigación y la extensión reconstruyendo, de este modo, el marco que sustenta los encuentros cotidianos de aprendizaje. (p. 27)

De esta definición, llaman la atención varios aspectos importantes de anotar. En primer lugar, se evidencia que para estos autores, el estudiantado y el personal docente son los que construyen el currículo, a partir de sus experiencias, sus vivencias y el contexto en el que se desenvuelven. Con base en esto, se afirma que un currículo así hará que la educación tenga un gran impacto y sea significativa para la vida de las personas.

En segundo lugar, estos autores hacen alusión a la investigación y a las experiencias cotidianas como parte del currículo. Sin duda alguna, estos dos aspectos enriquecen los procesos curriculares, tal como lo plantean Ford, Davern y Schnorr (1999), y le dan sentido al currículo. En este punto cabe señalar que la investigación, no es una función extra de las y los docentes, sino que es inherente a su labor; por tal razón, resulta fundamental que sea considerada como tal, para poder, así, contribuir a la transformación de la educación, a partir del diseño curricular.

Posner (1998) señala que ofrecer una definición de currículo no es una tarea simple. Esto es comprensible, ya que como se ha visto hasta el momento, no existe una definición universal de este término, sino que existe una amplia gama de ellas, que en algunos aspectos difieren y en otros se asemejan. Este autor hace referencia a "seis conceptos comunes de currículo" (p. 11), los cuales sirven de base para entender que con el paso del tiempo, este concepto ha ido cambiando poco a poco.

Posner (1998) señala, en primer lugar, que el currículo ha sido concebido como los objetivos que deben cumplirse en una materia determinada. En segundo lugar, agrega que el currículo ha sido entendido, también, como el plan de un curso específico. Se puede decir, que esta concepción es común, aunque bastante reducida y limitada, ya que sólo se centra en el plan de un curso determinado. La tercera forma 
en la que se ha comprendido el currículo, según este autor, consiste en verlo como una serie de temas organizados. La cuarta sería el conjunto de materiales utilizados en una clase. Además, el autor precisa que el currículo también se ha definido como un plan de estudios, constituido por una variedad de cursos que la población estudiantil debe aprobar. Finalmente, indica que otra conceptuación de currículo, es la que hace referencia al conjunto de experiencias que tiene el estudiantado y que son planificadas por la escuela.

Como puede notarse, la mayoría de estas definiciones, a excepción de la última, se centran en aspectos como contenidos, materias, objetivos, resultados y productos. Es evidente que estas definiciones no toman en cuenta a los diferentes participantes del proceso educativo (ni sus experiencias, ni sus vivencias), sino que parten de lo que se espera de ellos, de los resultados en términos de contenidos, conceptos o materias.

Podría decirse, entonces, que los aspectos tomados en cuenta en las definiciones planteadas por Posner (1998), son tan sólo algunos de los que forman parte del currículo. Sería muy reduccionista, concebir al currículo delimitado únicamente en uno de estos aspectos, pues como bien lo plantea el autor no se puede afirmar que exista una única cosa que pueda ser considerada como currículo. Los conceptos que menciona Posner sirven de base para comprender que el currículo no puede ser reducido únicamente a programas, contenidos, temas o asignaturas, sino que va mucho más allá, se trata de un término amplio que, de acuerdo con el MEP (2009),

(...) se sustenta en la interrelación de sus diferentes componentes, lo que implica comprender las relaciones que se establecen entre la institución educativa y la comunidad, la legislación actual, los programas de estudio, las metodologías, los recursos, el contexto escolar, comunal y global, el cuerpo docente, el estudiantado y la familia que demandan de un proyecto educativo construido con la participación de todas las instancias educativas. (p. 31-32)

Precisamente, tanto en la definición del MEP (2009), así como en las conceptualizaciones planteadas con anterioridad por Bolaños y Molina (2001), Peralta (2007) y Quesada et al. (2001), salta a la vista esa expansión, por decirlo de alguna manera, del término currículo, al hacer mención a aspectos como experiencias, vivencias, contextos, procesos de aprendizaje, entre otros; por esto, es que Grundy (1998) menciona que el currículo es "una construcción social" (p. 39). De este modo, una vez más, es evidente que el concepto de currículo es amplio, ya que implica toda una realidad educativa y los diferentes aspectos que forman parte de ella.

Además de las definiciones anteriores, Castillejo (1992) de manera más específica, menciona que el currículo “ (...) es un 'proyecto'(...) vinculado a la realidad” (p.15). Definitivamente, el vínculo con la realidad, podría considerarse como un requisito fundamental del currículo, para lograr, de esa forma, procesos educativos útiles y con significado.

Este autor, también, destaca que el currículo tiene una “ (...) condición de instrumento pedagógico" (p. 15). En otras palabras, podría afirmarse que el currículo tiene una gran utilidad en el ámbito pedagógico y, por ende, en el de la educación. En relación con esto, Castillejo (1992) señala que el currículo, a causa de esa condición pedagógica que se citaba anteriormente, tiene tres ejes básicos, a partir de los cuales se resuelve. El primer eje consiste en los objetivos o finalidades, las cuales guían el currículo; el segundo, se centra en el individuo como tal, a quien va dirigido el currículo y, el tercer eje se basa en el marco que delimita el currículo y que hace que éste se pueda llevar a la práctica. Con base en estos tres ejes, Castillejo plantea que:

Integrando los tres ejes centrales o básicos que conforman el curriculum podemos entenderlo como una oferta o proyecto educativo que determina qué, cómo y con qué debe 
desarrollarse el proceso educativo. Es decir, cumple la función de especificar y justificar qué debe ser enseñado, a quién, bajo qué normas y condiciones, por quién, etc.

Por ello desde un prisma pedagógico (científico) podemos decir que el curriculum es la expresión racional y organizada de los elementos que integran el proceso educativo en un nivel, período, etc., determinado. (p. 16)

Se percibe, entonces, que en el currículo se deben tomar en cuenta todas las condiciones y los requerimientos necesarios para educar a las personas. De lo anterior, también, se desprende, que la determinación del currículo depende de los elementos que lo conforman. Por tanto, no solamente se afirma que no existe una definición universal, sino que cada currículo es diferente, ya que cada lugar en donde éste se desarrolla, tiene características particulares y los sujetos que participan en él, también tienen características propias que los diferencian entre sí y de otros grupos de individuos.

Para concluir con la conceptuación del currículo, cabe destacar el planteamiento de Pérez (1994) quien menciona que definir el término de currículo resulta ser muy complejo y que para definirlo es fundamental, también, tomar en cuenta una serie de elementos que intervienen en las prácticas educativas. A modo de síntesis, se puede decir, entonces, que el currículo está vinculado con la educación y está basado en la realidad en la que éste se cirncuscribe, de ahí la importancia de plantear currículos pertinentes y significativos para todas las personas que van a participar en su puesta en práctica.

Ahora bien, para poder comprender el currículo de una mejor manera, es fundamental hacer referencia a sus elementos. En este sentido, Peralta (2007) indica que teniendo claro que "todo currículo integra un 'conjunto de factores y elementos básicos', que son los que permiten su operacionalización en términos de favorecer estas vivencias y aprendizajes que se desean, aparece como fundamental identificar con mayor detalle cada uno de ellos" (p. 35).

Así, la autora plantea una serie de factores y elementos que constituyen el currículo. En la Figura 1 se sintetizan estos aspectos.

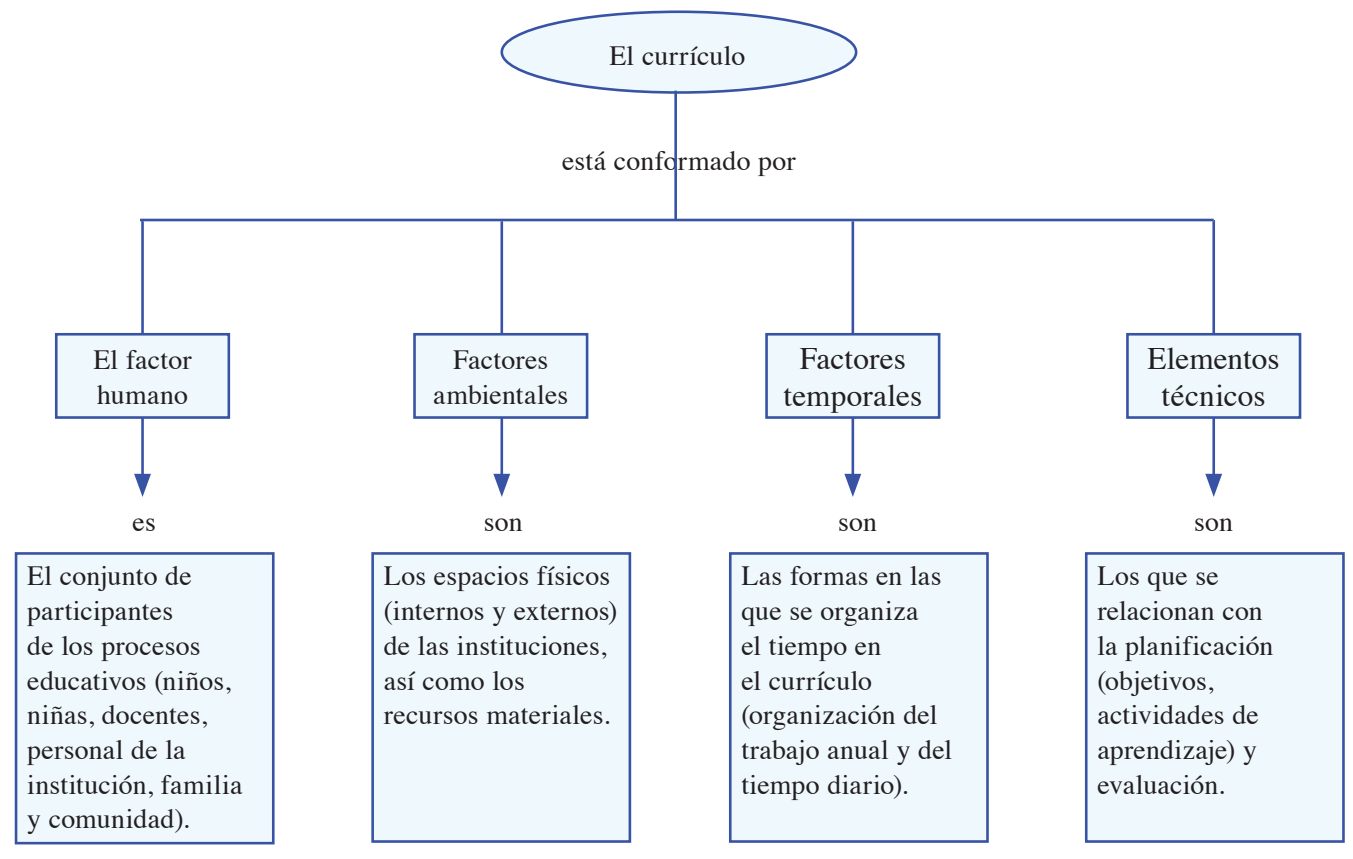

Figura 1. Factores y elementos del currículo.

Nota. Elaboración propia, a partir de Peralta, (1988). 
Se evidencia, entonces, que en el currículo interviene una serie de aspectos que son los que forman parte de la realidad educativa. Cabe destacar que estos factores y elementos detallados por Peralta (2007) se complementan, se integran entre sí y hacen posible el desarrollo del currículo.

Si se delimitan los aspectos ya detallados, a la realidad educativa costarricense y, específicamente, a los procesos educativos que se dan en el Ciclo Materno Infantil (CMI), es necesario hacer referencia a los elementos del currículo que destaca el MEP (2008). En este sentido, se plantea que los elementos del currículo son "espacios de interacción, jornada de trabajo, equipo y material didáctico, rol asumido por las niñas y los niños, rol asumido por la docente, integración de la familia y la comunidad, planeamiento de aula y registros de observación" (p. 14).

Puede apreciarse, claramente, que los factores y los elementos destacados por Peralta (2007), coinciden con los elementos del currículo del Ciclo Materno Infantil. Todos estos aspectos adquieren relevancia en las prácticas educativas, y son los que permiten que éstas sean de calidad y tengan significado para sus participantes (MEP, 2008).

De este modo, se ha hecho un breve repaso de los diferentes elementos del currículo, los cuales lo construyen y lo enriquecen. Estos planteamientos, junto con las conceptuaciones anteriores, son la base para poder hacer referencia al currículo preescolar costarricense, específicamente al relacionado con el Ciclo Materno Infantil (CMI).

\section{El currículo preescolar costarricense}

Por ser parte de una realidad sociocultural específica, el currículo preescolar costarricense tiene características que lo determinan como tal. Estas características, se encuentran plasmadas en algunos documentos del MEP, el cual es el ente encargado de dar la guía curricular que se debe poner en práctica en los jardines infantiles del sistema educativo público costarricense.

Para hacer referencia a estas características, cabe rescatar los planteamientos de Cerdas y Mata (2001), quienes precisan que el currículo preescolar de Costa Rica, pretende no ser tradicional, porque tiene un fundamento teórico-curricular humanista, pues busca el desarrollo integral de los sujetos, está acorde con el ambiente en el que éstos se desenvuelven y promueve la construcción de conocimientos por medio del constructivismo. Se evidencia, entonces, que este currículo, cumple con el objetivo de promover el desarrollo integral y de hacer que el proceso educativo resulte significativo para los niños y las niñas y, en general, para todos los que intervienen en él.

Desde esta perspectiva, se propone una visión dinámica del ser humano, a partir de la cual el currículo, se constitutuye en una valiosa propuesta integral e integrada, abierta, flexible, significativa y culturalmente pertinente, que retoma las experiencias de juego espontáneo y natural donde se propicia la acción física, mental social y emocional del sujeto con el ambiente que le rodea.

Dicha conceptualización integra como protagonistas activos al niño, la niña, la docente, la directora, el director, la familia, la comunidad y la asesora, quienes en sus interacciones definen la direccion del curriculum que se desarrolla (Cerdas y Mata, 2001, p. 7).

A manera de resumen, se puede anotar que el currículo preescolar costarricense pretende tener sentido, pues parte de la realidad en la que se encuentra inmerso y ubica a todos sus participantes como centro y punto de partida de él. Igualmente, este currículo intenta contribuir al desarrollo de todas las áreas del ser humano y promover la participación directa en el proceso educativo, 
de los diversos actores que intervienen en él. Asimismo, busca la construcción de conocimientos, más que la memorización de éstos, y toma en cuenta, tanto los resultados del proceso educativo, como el proceso como tal.

Dentro de las características del currículo preescolar costarricense, se puede hacer referencia también a la estructura técnico-curricular de la Educación Preescolar costarricense, según el MEP (2002). En general, de acuerdo con el MEP, la Educación Preescolar se divide en dos ciclos: Materno Infantil y Transición.

Despues de haber aclarado lo anterior, es necesario hacer referencia específica al CMI como parte del sistema educativo costarricense.

\section{El currículo preescolar costarricense del Ciclo Materno Infantil}

Según el MEP (2001), el “ (...) Ciclo Materno Infantil conjuntamente con el Ciclo de Transición constituyen la estructura técnica curricular de la Educación Preescolar que corresponde al primer nivel del Sistema Educativo Costarricense" (p. 45). Podría decirse, entonces, que el CMI al estar antes del Ciclo de Transición, constituye la base de la educación costarricense.

Esta misma fuente destaca que, específicamente, el CMI “ (...) es aquel que ofrece un servicio educativo de calidad, con un fuerte componente pedagógico, dirigido a niños y niñas en edades comprendidas desde el nacimiento hasta su ingreso al Ciclo de Transición” (p. 45). Se puede ver, que el CMI tiene el propósito de educar a la población infantil menor de cinco años y medio, y no de convertirse en una guardería. En cuanto a esto, el MEP postula lo siguiente.

El servicio educativo que se brinda en el Ciclo Materno Infantil no debe limitarse a 'cuidar niños' y a la 'academización' de sus actividades, o bien, a simplificar el Programa del Ciclo de Transición (1996). De proceder así, la consecuencia es el desarrollo de los procesos de aprendizaje temporales, ficticios que no se convierten en parte de los individuos, sino en algo que hay que aprender para satisfacer a otro, llámese padre, madre, encargado o maestra. (p. 46)

A lo anterior, el MEP (2001) añade que la prioridad fundamental de este Ciclo, consiste en la “ (...) atención a las necesidades, potencialidades e intereses de niños y niñas" (p. 45). En otras palabras, este Ciclo intenta contribuir al desarrollo integral de los infantes, tomando en cuenta lo que son, lo que les interesa y lo que requieren.

Ahora bien, teniendo claro qué es el CMI y qué es lo que pretende, resulta importante, también, explicar a cuál población infantil es a la que va dirigido, así como cuál es la forma en que éste se organiza, para atender a estos niños y niñas. El MEP (2001) propone que a causa de la diversidad de población a la que responde este Ciclo, es necesario, para fines didácticos, exclusivamente, establecer una organización de grupos, según la edad de los niños y las niñas que la conforman. De este modo, se establecen tres grandes grupos: el grupo de bebés, el de maternal y el interactivo.

A la vez, estos grupos están subdivididos de la siguiente forma: a) el grupo de los bebés, se subdivide en bebés I, constituido por niños y niñas de cero a seis meses y en bebés II, constituido por infantes de seis a doce meses; b) el grupo de maternal, se subdivide en maternal I, que atiende párvulos de un año hasta los dos años y seis meses y en maternal II, con niños y niñas de dos años y seis meses hasta los tres años y seis meses y c) el grupo interactivo, se subdivide en interactivo I, con una población infantil de tres años y seis meses, hasta los cuatro años y seis meses e interactivo II, constituido por infantes de cuatro años y seis meses hasta que ingresan al Ciclo de Transición. 
Es importante señalar que, en Costa Rica, en el sistema educativo público, únicamente están funcionando los grupos interactivos II del CMI, por lo que niños y niñas menores, son atendidos por otras instancias (CEN-CINAI, por ejemplo). En relación con esto, cabe anotar que “(...) se espera que el ingreso de los niños y las niñas a este Ciclo, se realice lo más tardíamente posible, con el propósito de permitir al bebé y su familia construir y fortalecer los vínculos afectivos antes de aventurarse a la relación con extraños" (MEP, 2001, p. 45).

Por otra parte, es preciso aclarar que aunque la edad que se establece para entrar a este grupo es de cuatro años y seis meses, según el MEP (2002) en la reforma del primer artículo del Decreto $\mathrm{N}^{\circ} 27845$ de 1999, se afirma que si el centro educativo tiene capacidad, puede matricular a niños y niñas de cuatro años y tres meses para que ingresen al grupo interactivo II.

Ahora bien, el MEP elaboró un Programa de Estudio específicamente para este Ciclo, en el que se encuentran, de una forma más amplia, las ideas que se han planteado hasta el momento. Es necesario, que las personas profesionales en educación preescolar, aun las que atienden el Ciclo de Transición reciban capacitación sobre dicho Programa, pero, además, por sí mismas deben tener la iniciativa de conocer este Programa, analizarlo y empoderarse de él, con el fin de propiciar una educación de calidad para niños y niñas menores de 6 años. Sin duda alguna, el Programa como tal, no puede tener un impacto en la educación costarricense, si las docentes de preescolar, aunque lo tengan, no hacen lo posible por lograr lo anterior. Se considera que este Programa es una herramienta que promueve cambios significativos en la educación preescolar, pero depende de cada docente y de los diferentes participantes de los procesos educativos del nivel preescolar, considerarlo como tal y asumir la responsabilidad de conocerlo a profundidad, para poder llevarlo a la práctica.

Después de tener claro qué es el CMI, a quién va dirigido y cuál es la organización de sus grupos, se considera fundamental, hacer un breve recorrido por el Programa de Estudio del Ciclo Materno Infantil (PECMI), como una manera de brindar un acercamiento a este currículo preescolar del sistema costarricense.

\section{Programa de Estudio del Ciclo Materno Infantil}

En primer lugar, se puede decir que el PECMI, según el MEP (2002), fue aprobado por el Consejo Superior de Educación en la Sesión No10-2000 del 29 de febrero del año 2000. En general, el PECMI se divide en cinco apartados, los cuales son precedidos por una presentación y una justificación del Programa. A continuación, se explican estos apartados, aclarando que lo que se pretende es brindar una aproximación al PECMI e invitar al lector a conocer, con mayor detalle, el documento completo.

El primer apartado del PECMI hace referencia a los fundamentos jurídicos y teórico-curriculares que se plantean en este Programa. Se destacan los fundamentos de la educación preescolar, los principios del desarrollo infantil, las características de los niños y las niñas de cero a cinco años, así como algunos rasgos personales y profesionales que deben tener las docentes que atienden el CMI.

Este apartado resulta fundamental porque, precisamente, las prácticas docentes, deben partir de los planteamientos generales de un país, con respecto a la educación y, sin duda alguna, las y los docentes deben conocer cuáles son estos planteamientos. Asimismo, es necesario que las personas profesionales en educación conozcan cuáles son las características de las poblaciones infantiles con las que están trabajando; en este caso, las docentes de educación preescolar deben tener claridad sobre cómo es, en términos generales, el desarrollo integral de los niños y las niñas, con el fin de construir procesos pedagógicos significativos, útiles y relevantes. 
En el segundo apartado del PECMI, se explica qué es el CMI y se aclara que el fin de éste es "(...) el desarrollo integral del niño y la niña, en cuanto a sus intereses, necesidades, características, potencialidades y talentos (p. 46). También se explicita que en el “(...) Ciclo Materno Infantil, la población meta estará constituida por todos los niños y niñas en edad de 0 a 5 años, es decir, 420.000 infantes, según proyecciones de MIDEPLAN correspondientes al período 2000-2015" (p. 46).

Lo que se refiere al desarrollo didáctico y metodológico, se evidencia en la tercera parte del Programa. Precisamente, aquí se define lo que es el currículo y se explican los cuatro propósitos que guían el trabajo de las docentes. Estos cuatro propósitos, según el MEP (2001) “ (...) están organizados a partir de cuatro núcleos generadores, en los cuales se destacan los siguientes componentes: el sociocultural, el personal, los conocimientos y la comunicación” (p. 54).

El MEP (2001) argumenta que estos propósitos del PECMI, se complementan, se integran y no constituyen agrupaciones que se excluyan entre sí. Cabe señalar que dentro de este Programa, en los cuatro propósitos que se mencionan, se destaca el papel del niño y la niña, la docente, la familia y la comunidad. Por tanto, puede afirmarse que el PECMI busca que, en los procesos educativos dirigidos a la población infantil menor de cinco años y medio, intervengan, de manera real y activa, las diferentes personas que forman parte de la vida de los niños y las niñas. La relación familia-comunidad-centro educativo es básica para poder lograr un desarrollo integral, tal como se evidencia en los propósitos que se encuentran en el Programa.

Precisamente, Peralta (2002) argumenta que uno de los criterios de calidad de la educación inicial es el criterio de integralidad. Al respecto, la autora considera lo siguiente:

Plantear el criterio de integralidad, supone tener presente las dos dimensiones que puede asumir: por una parte, que toda acción educativa debe abarcar e integrar los diversos aspectos del desarrollo y aprendizaje del educando, ya que así actúa y el niño/la niña se vincula con su entorno; por otra evidencia que para ello es necesario la participación de diferentes agentes e instancias formativas en los diferentes contextos en los que participa el niño/la niña, ya que a través de ellos se va a posibilitar esa vinculación con lo real, que no se puede obtener de la acción aislada de un miembro de la sociedad. (p. 228)

En el caso del PECMI, este criterio se ve reflejado, ya que los propósitos que están establecidos implican una integralidad de las áreas del desarrollo humano y, a la vez, una integralidad entre los diferentes actores que intervienen en el proceso educativo. De ahí la gran importancia de que las personas profesionales en educación preescolar, tengan claridad de los alcances que tienen estos cuatro propósitos.

Además de los propósitos, en el tercer apartado del PECMI, se habla acerca de las diferentes maneras en que se pueden organizar los grupos, es decir, se hace referencia a la organización horizontal, vertical o mixta del estudiantado, y acerca de cómo debe ser el espacio, en general, en el que se pone en práctica el currículo del CMI. Asimismo, se hace alusión a los espacios de interacción que deben existir en el aula, a saber: el espacio para el recibimiento, para el sueño o descanso, para la alimentación, para la higiene y el aseo personal, para el archivo y la exposición y, por último, el espacio para el juego. Es importante anotar, que el espacio para el juego incluye otros espacios: para el movimiento, para juegos que desarrollan la actividad perceptiva, para la comunicación y la expresión, para la identificación y la representación, así como para la cría de animales y el cultivo de plantas.

Puede decirse que estos espacios de interacción, responden a las características, los intereses y las necesidades de la niñez a la que va dirigido el PECMI; por este motivo, no deben ser visualizados 
de forma separada, sino todo lo contrario, deben desarrollarse como espacios que tienen una estrecha relación entre sí. Específicamente, en este caso, depende de los y las profesionales en educación preescolar, implementar estos espacios de forma adecuada, para que realmente contribuyan al desarrollo integral de los niños y las niñas de edad preescolar.

Sumado a lo anterior, en este apartado del PECMI, se detalla cómo debe ser la interacción con el tiempo y la manera de organizarlo, así como algunas sugerencias sobre los materiales que los niños y las niñas pueden utilizar, según la edad que tengan. En este caso, es pertinente indicar que la organización del tiempo en la jornada diaria dependerá de la edad infantil con la que se esté trabajando; al respecto, el PECMI explicita diferentes opciones de jornada según los grupos de niños y niñas. No obstante, cabe anotar, también, que esta organización del tiempo va a estar delimitada por las situaciones particulares que puedan generarse en la jornada diaria $\mathrm{y}$, además, por los intereses de los infantes. Es evidente, de esta manera, que la organización del tiempo debe ser flexible y no rígida, pues de lo que se trata es de estructurar el tiempo de manera que se puedan cumplir con los propósitos establecidos en el programa.

Finalmente, en este tercer apartado, se mencionan algunos elementos importantes de la planificación, varias sugerencias didácticas acordes con los grupos de edad en los que se organiza el CMI y una propuesta con la que se intenta involucrar a la familia y la comunidad en el proceso educativo de este Ciclo. Salta a la vista, una vez más, la gran importancia que el PECMI le asigna a la familia y a la comunidad, pues se parte de la idea de que la educación y los procesos que en ella se generan, deben ser construidos por diferentes actores que participan en forma activa y dinámica, con el fin de propiciar el desarrollo integral de la niñez.

En el cuarto apartado del PECMI, se incluyen las referencias bibliográficas utilizadas en la elaboración del Programa, conjuntamente con la blibliografía que se puede consultar. Por último, en el quinto apartado, se añaden los anexos del programa, en los que se presentan algunos ejemplos de distribución del espacio institucional, de la organización del tiempo, de las guías para el planeamiento didáctico, de la evaluación del desempeño docente, de la evaluación del funcionamiento de la institución, de los registros de observación por grupo, de los instrumentos para entrevistar a madres, padres o encargados, así como un ejemplo de evaluación de los encuentros con la familia.

Es necesario destacar que este último apartado, constituye una herramienta que guía y enriquece el trabajo docente. Precisamente, por esta razón, es importante que sean considerados como recursos valiosos y necesarios que enriquecen y guían los procesos educativos preescolares.

En general, éstos son, en forma resumida, los apartados que conforman el PECMI. Como puede verse, dentro de este programa, se toman en cuenta todos los elementos del currículo, los cuales, como se explicó anteriormente, se encuentran en estrecha relación, con el fin de ofrecer a los niños y las niñas de cero a cinco años, una educación integral y significativa. Una vez más, es pertinente señalar que el PECMI propicia una visión de integralidad no solamente en cuanto al desarrollo infantil, sino que, también, en todo lo relacionado con el proceso educativo. De ahí la pertinencia y la relevancia de un Programa como éste en el sistema educativo de Costa Rica.

\section{Reflexiones finales}

Como bien se ha visto a lo largo del escrito, el currículo es un concepto muy amplio que trasciende una lista de contenidos o las cuatro paredes de un aula. El currículo implica la interrelación de una serie de elementos que lo construyen, lo transforman y le dan sentido al mismo. Precisamente, de ahí se desprende la importancia de que los involucrados en la educación, en 
especial los y las docentes, tengan una idea clara de qué es el currículo, qué es lo que pretende, qué elementos lo conforman y qué tipo de educación quiere lograr en la sociedad actual.

Para lograr lo anterior, es necesario que estos y estas profesionales asuman como inherente a su labor, la función investigativa y de actualización constante. A la luz del surgimiento del PECMI, es imprescindible que las docentes de preescolar se preocupen por conocer, analizar y encontrar el sentido que este programa tiene. No es justificable argumentar que este Programa no se conoce o que es complicado, por el hecho de que rompe el esquema del Programa del Ciclo de Transición y es completamente diferente a éste en cuanto a su estructura, su contenido y la población infantil a la que va dirigido.

El currículo del CMI, planteado en el PECMI pretende lograr una educación relevante y acorde con las características, los intereses y las necesidades de la población infantil menor de cinco años y medio. Por tal razón, se convierte en una guía útil e, inclusive, en una transformación positiva de los procesos educativos preescolares, dirigidos a los niños y niñas menores de cinco años y medio. Una vez más, salta a la vista la gran importancia que tienen las docentes de preescolar, para lograr esos cambios y esas transformaciones educativas, que toda sociedad requiere.

El Ciclo Materno Infantil es una opción curricular del sistema educativo público costarricense. La idea de este breve acercamiento al currículo del CMI es motivar a las docentes a acercarse a este Ciclo, a formularse preguntas, a cuestionarse y hasta a contrariarse. Solamente de esta forma se podrán lograr los propósitos planteados en este Programa, relacionados con el desarrollo integral de la población infantil y la participación conjunta de todos los actores del proceso educativo.

\section{Referencias Bibliográficas}

Bolaños, G. \& Molina, Z. (2001). Introducción al currículo. (2a. ed.). San José, Costa Rica: Editorial de la Universidad Estatal a Distancia.

Castillejo, J. (1992). El currículo en la educación infantil. En E. Martínez (Ed). El currículo de la educación preescolar. México, D.F.: Santillana.

Cerdas, A. \& Mata, A. (2001). Implementación Programa de Estudio para el Ciclo Materno Infantil. San José, Costa Rica: Ministerio de Educación Pública.

Costa Rica. Ministerio de Educación Pública. (2001). Programa de Estudio Ciclo Materno Infantil. San José, Costa Rica: Autor.

Costa Rica. Ministerio de Educación Pública. (2002). Educación preescolar en Costa Rica. Consideraciones y Lineamientos para el desarrollo de la práctica pedagógica en el nivel preescolar. San José, Costa Rica: Autor.

Costa Rica. Ministerio de Educación Pública. (2008). Concepción Curricular. Programa de Estudio Ciclo Materno Infantil. San José, Costa Rica: Autor.

Costa Rica. Ministerio de Educación Pública. (2009). Orientaciones Curriculares Nacionales. San José, Costa Rica: Autor. 
Chaves, A. (2004). Análisis de la congruencia entre el Programa de Estudio para el Ciclo Materno Infantil, del Ministerio de Educación Pública y las características, intereses y necesidades de los niños y las niñas de 4 años y medio a 5 años y medio, que asisten a los grupos interactivos II de dos jardines infantiles públicos del área metropolitana. Tesis para optar por el grado de Licenciatura en Pedagogía con énfasis en Educación Preescolar. Heredia, Costa Rica: Universidad Nacional.

D’Angelo, E. y Medina, A. (1999). Elección de materiales desde un enfoque didáctico comunicativo. En Materiales y recursos: La educación en los primeros años. (pp. 52-77). Buenos Aires, Argentina: Ediciones Novedades Educativas.

Ford, A., Davern, L. \& Schnorr, R. (1999). Educación inclusiva. "Dar sentido" al currículo. Aulas Inclusivas. Madrid; España: Narcea de Ediciones.

Grundy, S. (1998). Producto o praxis del curriculum. ( $3^{\mathrm{a}}$ ed). Madrid, España: Ediciones Morata.

Peralta, V. (2002). Una pedagogía de las oportunidades. Santiago, Chile: Editorial Andrés Bello.

Peralta, V. (2007). El currículo en el Jardín Infantil. Un análisis crítico. (1 ${ }^{\mathrm{a}}$ Reimp. de la $3^{\mathrm{a}}$ ed.) Santiago, Chile: Editorial Andrés Bello.

Pérez. R. (1994). El curriculum y sus componentes. Hacia un modelo integrador. Barcelona, España: Oikos-Tau.

Posner, G. (1998). Análisis del currículo. (2ª ed.). Bogotá, Colombia: Mc Graw-Hill.

Quesada, M., Cedeño, M. \& Zamora, J. (2001). El diseño curricular en los planes de estudio: aspectos teóricos y guía metodológica. Heredia, Costa Rica: Editorial de la Universidad Nacional. 